\title{
Efficient Use of PPM in Spectral-Amplitude-Coding Optical CDMA Systems
}

\author{
Hossam M. H. Shalaby, Senior Member, IEEE
}

\begin{abstract}
A prominent technique, for reducing the effect of phase-induced intensity noise (PIIN) in spectral-amplitude-coding optical code-division multiple-access (SAC-OCDMA) systems, is proposed. In this technique, each user is assigned two orthogonal code sequences, selected from a minimum cross-correlation code set. In addition, two-level $M$-ary pulse-position modulation ( $M P P M)$ scheme is adopted, where each level corresponds to a code sequence. This technique decreases the effect of PIIN and allows transmission at high data rates. The bit error rate (BER) of the proposed system is derived, taking into account the effects of PIIN, shot noise, and thermal noise. Two performance measures of this system, namely the BER and maximum achievable number of users, are compared to that of traditional systems adopting complementary and non-complementary keying. Our results reveal that the proposed technique allows each user to transmit at high data rates, compared to traditional techniques, while keeping the BER well below a prescribed threshold. Finally, our results are extended to higher levels of MPPM schemes with multicode modulation.
\end{abstract}

Index Terms-Bit-error-rate (BER), $M$-ary pulse-position modulation ( $M$ PPM), modified prime sequence (MPS) codes, modified quadratic congruence (MQC) codes, multicode modulation, optical code-division multiple access (OCDMA), spectral-amplitude coding (SAC).

\section{INTRODUCTION}

$\mathbf{S}$ PECTRAL-AMPLITUDE-CODING optical code-division multiple-access (SAC-OCDMA) [1], [2] is a promising, cost effective, multiple-access technique that can be used in future passive-optical networks (PONs), free-space optics (FSO), optical wireless communications, etc. [3]-[5]. However, it suffers from the effect of phase-induced intensity noise (PIIN), which seriously degrades the system performance and limits the number of simultaneous users that can be accommodated with reliable communications. Further, PIIN also limits the data transmission rates of each user, which is essential for future communications. Several attempts to reduce or mitigate the effects of PIIN have appeared in literature. One such attempts is to use $M$-ary pulse-position modulation (MPPM), which distributes its effects over several time slots [6]. In order to alleviate the side effect of increasing the system bandwidth

Manuscript received July 09, 2012; revised September 17, 2012; accepted September 20, 2012. Date of publication October 02, 2012; date of current version November 16, 2012.

The author is with the Department of Electronics and Communications Engineering, Egypt-Japan University of Science and Technology (E-JUST) Alexandria 21934, Egypt, on leave from the Electrical Engineering Department, Alexandria University, Alexandria 21544, Egypt (e-mail: shalaby@ieee.org).

Color versions of one or more of the figures in this paper are available online at http://ieeexplore.ieee.org.

Digital Object Identifier 10.1109/JLT.2012.2222017 when using MPPM, complementary keying has been adopted in [6]. Hadamard codes are good candidates to achieve complementary keying, where the cross-correlation between any two different code sequences is equal to the cross-correlation between any code sequence and the complement of another code sequence. Another attempt to reduce the effects of PIIN is to develop code sets with minimum fixed cross-corrections [7]. Examples of such codes are modified quadratic congruence (MQC) code [8], modified frequency-hopping (MFH) code [9], and balanced incomplete block design (BIBD) code [10]. Since the above cross-correlation property (equality to that of the complement) does not exist in this type of codes, one cannot use complementary keying and hence MPPM is not possible without increasing the system bandwidth. Unfortunately, when a user needs to transmit at high data rates the above solutions are not enough and cannot provide reliable communications with acceptable number of users.

In this paper, we propose an alternative, but equivalent, technique that combines both complementary keying and minimum cross-correlation property. Basically, we assign two orthogonal code sequences, with minimum cross-correlation, to each user and encode the data symbols from each user using a two-level $M P P M$ scheme. During modulation, each level would correspond to a different code sequence, which would still alleviate increasing the system bandwidth when using $M P P M$. The aforementioned codes (with fixed cross-correction) are not suitable here as they do not provide orthogonal sequences. One possible code that possesses both minimum cross-correlation and some orthogonal sequences is the modified prime sequence code [11]. In this code, code sequences are generated using a prime number $p$. Each code sequence has a weight $w=p$ and a length $L=p^{2}$. The code sequences can be divided into $p$ groups, each group consists of $p$ different code sequences. The cross-correlation between any two code sequences is zero (orthogonal) if the code sequences are selected from same group, whereas it is one if the code sequences are selected from two different groups.

Our proposed technique combines two advantages, namely decreasing the effect of PIIN and allowing transmission at high data rates without excessive increase in system bandwidth. We analyze the performance of the proposed system and derive an expression for the bit error rate (BER), taking into account the effects of PIIN, shot noise, and thermal noise. Next we numerically compare two performance measure of this system, namely the BER and maximum achievable number of users, with that of traditional systems adopting complementary and non-complementary keying. Our results reveal that the proposed technique is more power efficient than traditional ones and allows each user to transmit at higher data rates while keeping the BER well 
below a prescribed threshold. By assigning more-than-two orthogonal code sequences to each user, our results can be easily extended to multi-level MPPM scheme, where each level corresponds to a unique code sequence.

The remaining of this paper is organized as follows. The system description and the receiver model are presented in Section II. Section III is devoted for the derivation of the bit error probabilities of the proposed system, taking into account the effects of several noise sources. In Section IV we present some numerical results, where we investigate the effect of some design parameters (e.g., number of users, average power, code parameters, etc.) on the performance of the proposed scheme. Comparisons with corresponding systems adopting complementary and non-complementary keying are also presented in same section. Extension to higher levels of MPPM schemes with more-than-two orthogonal code sequences is studied in Section V. Finally, we give some concluding remarks in Section VI.

\section{SYSTEM DESCRIPTION}

In this section we describe our system model including both the transmitter and receiver. In addition, we introduce the the decision rule for the system.

\section{A. Transmitter Side}

We consider a synchronous SAC-OCDMA network which is composed of $N$ users (or information sources). The $n$th information source, $n \in\{1,2, \ldots, N\}$, generates iid $M$-ary data symbols or vectors $\left\{\boldsymbol{d}_{\boldsymbol{n}}\right\}$, where $\boldsymbol{d}_{\boldsymbol{n}}=\left[d_{n 0}, d_{n 1}, \ldots, d_{n, M-1}\right]$ such that $d_{n m} \in\{-1,1\}$ for some $m \in \mathcal{M} \stackrel{\text { def }}{=}\{0,1, \ldots, M-$ $1\}$ and $d_{n l}=0$ for any other $l \in \mathcal{M}-\{m\}$. This sequence modulates the position of incoherent light pulses, emitted from an ideal broadband light source. A light pulse is generated in slot $m \in \mathcal{M}$, out of $M$ possible slots, if $d_{n m} \neq 0$. The slot duration is denoted by $\tau$ and the symbol duration is denoted by $T_{s}=$ $M \tau$. In addition, each user is assigned two orthogonal code sequences of length $L=p^{2}$ and weight $w=p$, selected from same group of a modified-prime code $\operatorname{set} \mathcal{C}(L, w, \lambda)$, where $p$ is a prime number and $\lambda=1$ is the maximum cross-correlation of the code. For example, user $n, n \in\{1,2, \ldots, N\}$, is assigned code sequences $\boldsymbol{c}_{\boldsymbol{n}}=\left\{c_{n}(1), c_{n}(2), \ldots, c_{n}(L)\right\}$ and $\boldsymbol{c}_{\boldsymbol{n}}^{\prime}=\left\{c_{n}^{\prime}(1), c_{n}^{\prime}(2), \ldots, c_{n}^{\prime}(L)\right\}$, where $c_{n}(i), c_{n}^{\prime}(i) \in\{0,1\}$ and $c_{n}(i) c_{n}^{\prime}(i)=0$ for any $i \in\{1,2, \ldots, L\}$. Each code sequence is used to modulate the intensities of the spectral components of each emitted light pulse [1]-[8]. The modulation procedure for user $n$ is as follows: If for any $m \in \mathcal{M}, d_{n m}=1$, spectral code sequence $\boldsymbol{c}_{\mathbf{1}}$ is used for modulation. If $d_{n m}=-1$, spectral code sequence $\boldsymbol{c}_{1}^{\prime}$ is used. Otherwise, no pulse is transmitted. Since both positive and negative data are considered, we call this technique bipolar MPPM SAC-OCDMA. In our analysis below, we denote the optical source frequency and bandwidth by $f_{c}$ and $\Delta \nu$, respectively.

\section{B. Data Transmission Rate}

Assuming equi-probable data symbols, each occurs with probability $1 / 2 \mathrm{M}$, then the nominal transmission rate is given by

$$
R_{b}=\frac{\log (2 M)}{T_{s}}=\frac{\log (2 M)}{M \tau} \text { nat } / \mathrm{s}
$$

where the natural number is taken as the basis of the "log" function. For a bit duration of $T_{b}$ and binary PPM, this transmission rate is equal to $(\log 2) /\left(T_{b}\right)$ nat/s, which is exactly the same as the rate if on-off keying (OOK) was used, without bandwidth expansion. Of course if $M>2$, we still have some bandwidth increase. For example, for 4-PPM, the transmission rate is $(3 \log 2) /(4 \tau)$ nat/s, i.e., a bandwidth expansion factor of $(4) /(3)$. Of course, one can even remove the bandwidth increase for any $M \geq 2$, simply by increasing the modulation levels above two by assigning more than two orthogonal code sequences for each user. This leads to what is called multicode modulation. Multicode modulation has been proposed for 2D OCDMA [12], [13]. The price paid in multicode modulation, however, is the added complexity in both circuit implementation and decision rule.

\section{Code Properties and Number of Users}

The cross-correlation function between any two code sequences $n, m \in \mathcal{C}(L, w, \lambda)$ is given by [11]

$$
\begin{aligned}
& \sum_{i=1}^{L} c_{n}(i) c_{m}(i) \\
& \quad= \begin{cases}p ; & \text { if } n=m, \\
0 ; & \text { if } n \text { and } m \text { share the same group and } n \neq m, \\
1 ; & \text { if } n \text { and } m \text { are from different groups. }\end{cases}
\end{aligned}
$$

Since the total number of available code sequences in any group of $\mathcal{C}(L, w, \lambda)$ is $p$ and each user is assigned two code sequences, the total number of network subscribers cannot exceed $p\lfloor(p) /(2)\rfloor$. Here $\lfloor x\rfloor$ denotes the largest integer not greater than $x$. Out of this number we assume that there are $N$ active or simultaneous users and the remaining users are idle. Without loss of generality, we always assume that the first user is the desired user. Let the random variable $T$ represent the number of active users in the first group. For uniform code assignment, it is easy to check that the probability distribution of $T$, given that user 1 is always active, can be written as

$$
P_{T}(t)=\frac{\left(\begin{array}{c}
\left\lfloor\frac{p}{2}\right\rfloor-1 \\
t-1
\end{array}\right)\left(\begin{array}{c}
(p-1)\left\lfloor\frac{p}{2}\right\rfloor \\
N-t
\end{array}\right)}{\left(\begin{array}{c}
p\left\lfloor\frac{p}{2}\right\rfloor-1 \\
N-1
\end{array}\right)}
$$

where $t \in\left\{t_{\min }, t_{\min }+1, \ldots, t_{\max }\right\}$ and

$$
\begin{aligned}
t_{\min } & =\max \left\{1, N-(p-1)\left\lfloor\frac{p}{2}\right\rfloor\right\} \\
t_{\max } & =\min \left\{N,\left\lfloor\frac{p}{2}\right\rfloor\right\} .
\end{aligned}
$$

\section{Receiver Side}

The block diagram of the bipolar MPPM SAC-OCDMA receiver is shown in Fig. 1 for the desired user. A balanced detection scheme is used to reduce the effect of multiple-access interference (MAI) [1]-[8]. The upper branch of the balanced detector is used to detect the received spectral components that correspond to user's code sequence $\boldsymbol{c}_{\boldsymbol{1}}$. The lower branch, on 


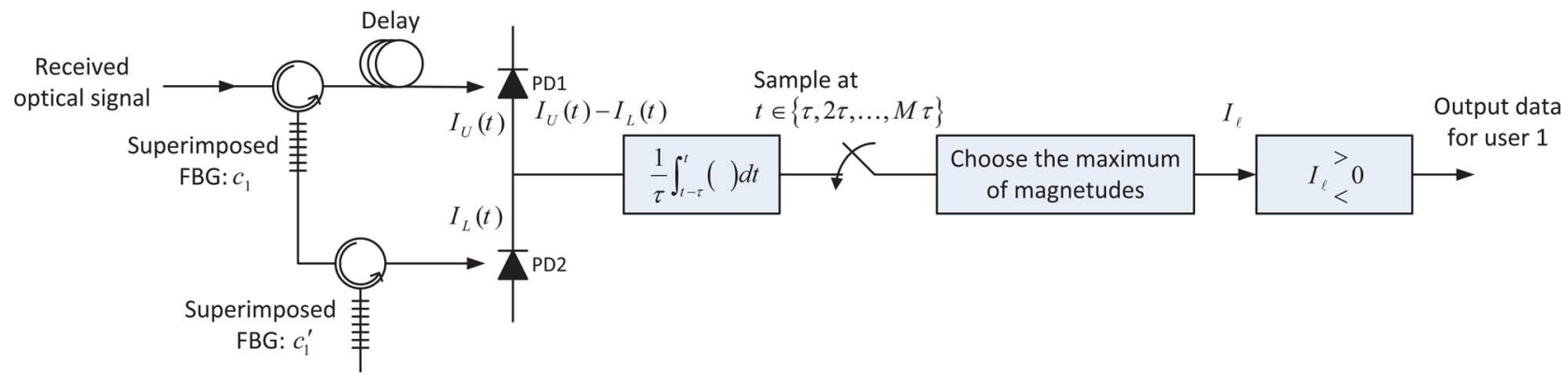

Fig. 1. Block diagram of a bipolar $M$ PPM SAC-OCDMA receiver.

the other hand, is used to detect the received spectral components that correspond to user's code sequence $\boldsymbol{c}_{1}^{\prime}$. Code detection can be implemented using superimposed fiber Bragg gratings (FBGs) [14], [15]. A superimposed FBG is written at same fiber location, which avoids delays between reflected spectral components. Both photodiodes' output currents are then subtracted and time averaged. The averaged current is then sampled at the end instants of each time slot $\{\tau, 2 \tau, \ldots, M \tau\}$ to give the set of decision currents $\left\{I_{0}, I_{1}, \ldots, I_{M-1}\right\}$. Finally this set is passed to the decision circuit.

\section{E. Decision Rule}

The decision current $I_{\ell}$ over slot $\ell \in \mathcal{M}$ is defined as $I_{\ell}=$ $I_{U, \ell}-I_{L, \ell}$, where $I_{U, \ell}$ and $I_{L, \ell}$ are the average output currents of the upper- and lower-branch photodiode, respectively. We define the decision rule as follows:

$$
\text { Decide } \begin{cases}\boldsymbol{b}^{\ell,+} ; & \text { if } I_{\ell}>\left|I_{m}\right| \quad \forall m \in \mathcal{M}-\{\ell\}, \\ \boldsymbol{b}^{\ell,-} ; & \text { if } I_{\ell}<-\left|I_{m}\right| \quad \forall m \in \mathcal{M}-\{\ell\},\end{cases}
$$

where $\boldsymbol{b}^{m, \pm}=\left[b_{0}^{m}, b_{1}^{m}, \ldots, b_{M-1}^{m}\right]$ with $b_{m}^{m}= \pm 1$ and $b_{i}^{m}=0$ for any $i \in \mathcal{M}-\{m\}$.

\section{TheORETICAL ANALYSIS}

In this section we derive the statistics of both upper- and lower-branch currents, as well as the statistics of the decision current. In addition, we develop an expression for evaluating the BER of the system.

\section{A. Statistics of Upper-Branch Current}

The resultant upper-branch incident field on the $\ell$ th slot, $\ell \in$ $\mathcal{M}$, can be written as

$$
\begin{aligned}
E_{U, \ell}(t)= & \sqrt{\frac{P_{0}}{L}} \sum_{n=1}^{N} \sum_{i=1}^{L} \\
& \times\left[\frac{\left|d_{n \ell}\right|+d_{n \ell}}{2} c_{n}(i)+\frac{\left|d_{n \ell}\right|-d_{n \ell}}{2} c_{n}^{\prime}(i)\right] \\
& \times g\left(\frac{t-\ell \tau}{\tau}\right) c_{1}(i) e^{j\left[\omega_{0} t+i \delta \omega t+\phi_{n i}(t)\right]}
\end{aligned}
$$

where $P_{0}$ is the peak received single-user power, $\delta \omega=$ $2 \pi \Delta \nu / L, f_{0}=f_{c}-\Delta \nu / 2-\Delta \nu / L, g(t)$ is a normalized envelope of a single-user light field of spectral width $\Delta \nu / L$, and $\phi_{n i}(t), n \in\{1,2, \ldots, N\}, i \in\{1,2, \ldots, L\}$, is the phase of the $i$ th chip optical signal of the $n$th user, assumed to be a Wiener process [16], [18]. For the sake of simplicity, we assume a rectangular envelope:

$$
g(t)= \begin{cases}1 ; & \text { if } t \in[0,1] \\ 0 ; & \text { otherwise }\end{cases}
$$

If the photodiode responsivity is denoted by $\mathcal{R}$, then the average $\ell$ th slot current of PD1 is given by

$$
I_{U, \ell}=\frac{\mathcal{R}}{\tau} \int_{\ell \tau}^{(\ell+1) \tau}\left|E_{U, \ell}(t)\right|^{2} d t .
$$

Using (6) and (8), and noticing that the cross-terms cancel out because of the averaging process (as long as $\tau>L / \Delta \nu$ ), we get:

$$
\begin{aligned}
I_{U, \ell}= & \frac{\mathcal{R} P_{0}}{L} \sum_{i=1}^{L} \frac{1}{\tau} \\
& \times \int_{\ell \tau}^{(\ell+1) \tau} g^{2}\left(\frac{t-\ell \tau}{\tau}\right) \mid \sum_{n=1}^{N}\left[\frac{\left|d_{n \ell}\right|+d_{n \ell}}{2} c_{n}(i)\right. \\
& \left.+\frac{\left|d_{n \ell}\right|-d_{n \ell}}{2} c_{n}^{\prime}(i)\right]\left.c_{1}(i) e^{j \phi_{n i}(t)}\right|^{2} d t .
\end{aligned}
$$

Expanding the last equation, we get

$$
\begin{aligned}
I_{U, \ell}= & \frac{\mathcal{R} P_{0}}{L} \sum_{i=1}^{L}\left\{\sum _ { n = 1 } ^ { N } \left[\frac{\left|d_{n \ell}\right|+d_{n \ell}}{2} c_{n}(i)\right.\right. \\
& \left.+\frac{\left|d_{n \ell}\right|-d_{n \ell}}{2} c_{n}^{\prime}(i)\right] c_{1}(i) \\
& +2 \sum_{n=1}^{N-1} \sum_{m=n+1}^{N}\left[\frac{\left|d_{n \ell}\right|+d_{n \ell}}{2} c_{n}(i)\right. \\
& \left.+\frac{\left|d_{n \ell}\right|-d_{n \ell}}{2} c_{n}^{\prime}(i)\right] \\
& \times\left[\frac{\left|d_{m \ell}\right|+d_{m \ell}}{2} c_{m}(i)\right. \\
& \left.+\frac{\left|d_{m \ell}\right|-d_{m \ell}}{2} c_{m}^{\prime}(i)\right] c_{1}(i) \\
& \times \frac{1}{\tau} \int_{\ell \tau}^{(\ell+1) \tau} g^{2}\left(\frac{t-\ell \tau}{\tau}\right) \\
& \left.\times \cos \left(\phi_{n i}(t)-\phi_{m i}(t)\right) d t\right\} .
\end{aligned}
$$


Making use of the properties of $\mathcal{C}(L, w, \lambda)$ code and assuming that the number of active users in group 1 is $t$, we get

$$
\begin{aligned}
I_{U, \ell}= & \frac{\mathcal{R} P_{0}}{L}\left\{w \cdot \frac{\left|d_{1 \ell}\right|+d_{1 \ell}}{2}+\lambda \kappa_{\ell}\right. \\
& +2 \sum_{n=1}^{N-1} \sum_{m=n+1}^{N} \sum_{i=1}^{L}\left[\frac{\left|d_{n \ell}\right|+d_{n \ell}}{2}\right. \\
& \times \frac{\left|d_{m \ell}\right|+d_{m \ell}}{2} c_{n}(i) c_{m}(i) c_{1}(i) \\
& +\frac{\left|d_{n \ell}\right|+d_{n \ell}}{2} \frac{\left|d_{m \ell}\right|-d_{m \ell}}{2} c_{n}(i) c_{m}^{\prime}(i) c_{1}(i) \\
& +\frac{\left|d_{n \ell}\right|-d_{n \ell}}{2} \frac{\left|d_{m \ell}\right|+d_{m \ell}}{2} c_{n}^{\prime}(i) c_{m}(i) c_{1}(i) \\
& \left.+\frac{\left|d_{n \ell}\right|-d_{n \ell}}{2} \frac{\left|d_{m \ell}\right|-d_{m \ell}}{2} c_{n}^{\prime}(i) c_{m}^{\prime}(i) c_{1}(i)\right] \\
& \left.\times X_{n m}^{i}(\ell)\right\}
\end{aligned}
$$

where

$$
\begin{aligned}
& X_{n m}^{i}(\ell) \stackrel{\text { def }}{=} \frac{1}{\tau} \int_{\ell \tau}^{(\ell+1) \tau} g^{2}\left(\frac{t-\ell \tau}{\tau}\right) \\
& \times \cos \left(\phi_{n i}(t)-\phi_{m i}(t)\right) d t .
\end{aligned}
$$

Here we defined the interference random variable $\kappa_{m}$ for any $m \in \mathcal{M}$ as:

$$
\kappa_{m} \stackrel{\text { def }}{=} \sum_{n=t+1}^{N}\left|d_{n m}\right| .
$$

In addition, we define the interference random vector $\kappa \stackrel{\text { def }}{=}$ $\left[\kappa_{0}, \kappa_{1}, \ldots, \kappa_{M-1}\right]$. Of course $\kappa_{m}$ follows a conditional binomial distribution and $\boldsymbol{\kappa}$ follows a conditional multinomial distribution, given $T=t$ :

$$
\begin{aligned}
P_{\kappa_{m} \mid T}\left(l_{m} \mid t\right) & =\left(\begin{array}{c}
N-t \\
l_{m}
\end{array}\right)\left(\frac{1}{M}\right)^{l_{m}}\left(1-\frac{1}{M}\right)^{N-t-l_{m}} \\
P_{\boldsymbol{\kappa} T}(\boldsymbol{l} \mid t) & =\frac{(N-t) !}{l_{0} ! l_{1} ! l_{2} ! \ldots l_{M-1} !}\left(\frac{1}{M}\right)^{N-t}
\end{aligned}
$$

where $l_{m} \in\{0,1, \ldots, N-t\}$ and $\boldsymbol{l} \stackrel{\text { def }}{=}\left[l_{0}, l_{1}, \ldots, l_{M-1}\right]$, such that $\sum_{m=0}^{M-1} l_{m}=N-t$.

\section{B. Mean and Variance of the Upper-Branch Current}

Using [16]-[18], the average and variance of $X_{n m}^{i}(\ell)$ are

$$
\begin{aligned}
\mu_{X_{n m}^{i}(\ell)} & \stackrel{\text { def }}{=} E\left\{X_{n m}^{i}(\ell)\right\}=0 \\
\sigma_{X_{n m}^{i}(\ell)}^{2} \stackrel{\text { def }}{=} & \operatorname{var}\left\{X_{n m}^{i}(\ell)\right\} \\
= & \frac{1}{2 \tau^{2}} \int_{\ell \tau}^{(\ell+1) \tau} \int_{\ell \tau}^{(\ell+1) \tau} g^{2}\left(\frac{t-\ell \tau}{\tau}\right) g^{2}\left(\frac{s-\ell \tau}{\tau}\right) \\
& \times e^{-2 \frac{|t-s|}{\tau_{c}}} d t d s
\end{aligned}
$$

respectively, where $\tau_{c}=L / \Delta \nu$ is the coherence time. Performing the last integration, we obtain

$$
\begin{aligned}
\sigma_{X_{n m}^{i}(\ell)}^{2} & =\frac{\tau_{c}}{2 \tau}\left[1-\frac{\tau_{c}}{2 \tau}\left(1-e^{-2 \frac{\tau}{\tau_{c}}}\right)\right] \\
& \approx \frac{\tau_{c}}{2 \tau}=B_{e} \tau_{c}
\end{aligned}
$$

where $B_{e}=(1) /(2 \tau)$ is the receiver electrical bandwidth. We have used the practical assumption that $\tau_{c} \ll \tau$. To get the average and variance of $I_{U, \ell}$, we use (15) and (16) in (11), taking into account the independence of the random variables $X_{n m}^{i}(\ell)$ :

$$
\begin{aligned}
\mu_{I_{U, \ell}} \stackrel{\text { def }}{=} & E\left\{I_{U, \ell}\right\}=\frac{\mathcal{R} P_{0}}{L}\left[w \cdot \frac{\left|d_{1 \ell}\right|+d_{1 \ell}}{2}+\lambda \kappa_{\ell}\right] \\
\sigma_{I_{U, \ell}}^{2} \stackrel{\text { def }}{=} & \operatorname{var}\left\{I_{U, \ell}\right\} \\
= & 4 B_{e} \tau_{c}\left(\frac{\mathcal{R} P_{0}}{L}\right)^{2} \sum_{m=t+1}^{N} \sum_{i=1}^{L}\left[\frac{\left|d_{1 \ell}\right|+d_{1 \ell}}{2}\right. \\
& \times \frac{\left|d_{m \ell}\right|+d_{m \ell}}{2} c_{1}(i) c_{m}(i) \\
& \left.+\frac{\left|d_{1 \ell}\right|+d_{1 \ell}}{2} \frac{\left|d_{m \ell}\right|-d_{m \ell}}{2} c_{1}(i) c_{m}^{\prime}(i)\right] \\
& +4 B_{e} \tau_{c}\left(\frac{\mathcal{R} P_{0}}{L}\right)^{2} \sum_{n=t+1}^{N-1} \sum_{m=n+1}^{N} \sum_{i=1}^{L}\left[\frac{\left|d_{n \ell}\right|+d_{n \ell}}{2}\right. \\
& \times \frac{\left|d_{m \ell}\right|+d_{m \ell}}{2} c_{n}(i) c_{m}(i) c_{1}(i) \\
& +\frac{\left|d_{n \ell}\right|+d_{n \ell}}{2} \frac{\left|d_{m \ell}\right|-d_{m \ell}}{2} c_{n}(i) c_{m}^{\prime}(i) c_{1}(i) \\
& +\frac{\left|d_{n \ell}\right|-d_{n \ell}}{2} \frac{\left|d_{m \ell}\right|+d_{m \ell}}{2} c_{n}^{\prime}(i) c_{m}(i) c_{1}(i) \\
& \left.+\frac{\left|d_{n \ell}\right|-d_{n \ell}}{2} \frac{\left|d_{m \ell}\right|-d_{m \ell}}{2} c_{n}^{\prime}(i) c_{m}^{\prime}(i) c_{1}(i)\right]
\end{aligned}
$$

Making use of the properties of $\mathcal{C}(L, w, \lambda)$ code and noticing that the average of the sum of products of three different codes is equal to $\lambda w / L$, the last variance becomes:

$$
\begin{aligned}
\sigma_{I_{U, \ell}}^{2}= & 4 B_{e} \tau_{c}\left(\frac{\mathcal{R} P_{0}}{L}\right)^{2}\left[\lambda \cdot \frac{\left|d_{1 \ell}\right|+d_{1 \ell}}{2} \sum_{m=t+1}^{N}\left|d_{m \ell}\right|\right. \\
& \left.+\frac{\lambda w}{L} \sum_{n=t+1}^{N-1} \sum_{m=n+1}^{N}\left|d_{n \ell}\right|\left|d_{m \ell}\right|\right] \\
= & 4 B_{e} \tau_{c}\left(\frac{\mathcal{R} P_{0}}{L}\right)^{2} \\
& \times\left[\lambda \kappa_{\ell} \cdot \frac{\left|d_{1 \ell}\right|+d_{1 \ell}}{2}+\frac{\lambda w}{L} \cdot \frac{\kappa_{\ell}\left(\kappa_{\ell}-1\right)}{2}\right] .
\end{aligned}
$$

\section{Statistics of Lower-Branch Current}

The resultant lower-branch incident field on the $\ell$ th slot, $\ell \in$ $\mathcal{M}$, can be written as:

$$
\begin{aligned}
E_{L, \ell}(t)= & \sqrt{\frac{P_{0}}{L}} \sum_{n=1}^{N} \sum_{i=1}^{L} \\
& \times\left[\frac{\left|d_{n \ell}\right|+d_{n \ell}}{2} c_{n}(i)+\frac{\left|d_{n \ell}\right|-d_{n \ell}}{2} c_{n}^{\prime}(i)\right] \\
& \times g\left(\frac{t-\ell \tau}{\tau}\right) c_{1}^{\prime}(i) e^{j\left[\omega_{0} t+i \delta \omega t+\phi_{n i}(t)\right]} .
\end{aligned}
$$

It should be noticed that $E_{L, \ell}(t)$ is independent of $E_{U, \ell}(t)$, as the chips selected by $\left\{c_{1}^{\prime}(i)\right\}$ and $\left\{c_{1}(i)\right\}$ are disjoint. Following a similar analysis to what we did in the last section, we 
get the average and variance of lower-branch decision current $I_{L, \ell}$ as:

$$
\begin{aligned}
\mu_{I_{L, \ell}} \stackrel{\text { def }}{=} & E\left\{I_{L, \ell}\right\}=\frac{\mathcal{R} P_{0}}{L}\left[w \cdot \frac{\left|d_{1 \ell}\right|-d_{1 \ell}}{2}+\lambda \kappa_{\ell}\right] \\
\sigma_{I_{L, \ell}}^{2} \stackrel{\text { def }}{=} & \operatorname{var}\left\{I_{L, \ell}\right\}=4 B_{e} \tau_{c}\left(\frac{\mathcal{R} P_{0}}{L}\right)^{2} \\
& \times\left[\lambda \kappa_{\ell} \cdot \frac{\left|d_{1 \ell}\right|-d_{1 \ell}}{2}+\frac{\lambda w}{L} \cdot \frac{\kappa_{\ell}\left(\kappa_{\ell}-1\right)}{2}\right]
\end{aligned}
$$

respectively.

\section{Statistics of Decision Current}

From the discussion in the above section, the decision current $I_{\ell}=I_{U, \ell}-I_{L, \ell}$ over slot $\ell \in \mathcal{M}$, has the following mean and variance, given desired user data vector $\boldsymbol{d}_{\mathbf{1}}=\boldsymbol{b}=\left[b_{0}, b_{1}, \ldots, b_{M-1}\right]$ and interference vector $\boldsymbol{\kappa}=\boldsymbol{l}=\left[l_{0}, l_{1}, \ldots, l_{M-1}\right]$,

$$
\begin{aligned}
\mu_{\ell, \boldsymbol{b}} & =\frac{\mathcal{R} P_{0}}{L} w b_{\ell} \\
\sigma_{\ell, \boldsymbol{b}, \boldsymbol{l}}^{2} & =\sigma_{\mathrm{PIIN} \mid \ell, \boldsymbol{b}, \boldsymbol{l}}^{2}+\sigma_{s \mid \ell, \boldsymbol{b}, \boldsymbol{l}}^{2}+\sigma_{T}^{2}
\end{aligned}
$$

respectively, where $\sigma_{\mathrm{PIIN} \mid \ell . \boldsymbol{b}, \boldsymbol{l}}^{2}, \sigma_{s \mid \ell, \boldsymbol{b}, \boldsymbol{l},}^{2}$, and $\sigma_{T}^{2}$ are the variances of the phase-induced intensity noise, shot noise, and thermal noise, respectively. These are given by

$$
\begin{aligned}
\sigma_{\mathrm{PIIN} \mid \ell, \boldsymbol{b}, \boldsymbol{l}}^{2} & =\sigma_{I_{U \mid \ell}, \boldsymbol{b}, \boldsymbol{l}}^{2}+\sigma_{I_{L \mid \ell, \boldsymbol{b}} \boldsymbol{l},}^{2} \\
& =4 B_{e} \tau_{c}\left(\frac{\mathcal{R} P_{0}}{L}\right)^{2} l_{\ell}\left[\lambda\left|b_{\ell}\right|+\frac{\lambda w}{L}\left(l_{\ell}-1\right)\right] \\
\sigma_{s \mid \ell, \boldsymbol{b}, \boldsymbol{l}}^{2} & =2 e B_{e}\left(\mu_{I_{U \mid \ell}, \boldsymbol{b} \boldsymbol{l},}+\mu_{I_{L \mid \ell, \boldsymbol{b}, \boldsymbol{l}}}\right) \\
& =2 e B_{e} \frac{\mathcal{R} P_{0}}{L}\left(w\left|b_{\ell}\right|+2 \lambda l_{\ell}\right) \\
\sigma_{T}^{2} & =\frac{4 k_{B} T^{\circ} B_{e}}{R_{L}}
\end{aligned}
$$

respectively. Here $e=1.6 \times 10^{-19} \mathrm{C}$ is the electron charge, $k_{B}=1.38 \times 10^{-23} \mathrm{~J} / \mathrm{K}$ is Boltzmann's constant, $T^{\circ}$ is the receiver noise temperature, and $R_{L}$ is the receiver load resistance.

\section{E. BER Evaluation}

The average symbol error rate (SER) $P_{e}$ can be written as

$$
\begin{aligned}
P_{e}=\frac{1}{2 M} \sum_{t=t_{\min }} & \sum_{\boldsymbol{l} \in \mathcal{X}} \sum_{m=0}^{M-1}\left[P_{e \mid \boldsymbol{d}_{1}, \boldsymbol{\kappa}, T}\left(\boldsymbol{b}^{m,+}, \boldsymbol{l}, t\right)\right. \\
+ & \left.P_{e \mid \boldsymbol{d}_{1}, \boldsymbol{\kappa}, T}\left(\boldsymbol{b}^{m,-}, \boldsymbol{l}, t\right)\right] P_{\boldsymbol{\kappa} \mid T}(\boldsymbol{l} \mid t) P_{T}(t),
\end{aligned}
$$

where the set $\mathcal{X} \stackrel{\text { def }}{=}\left\{\boldsymbol{l}=\left[l_{0}, l_{1}, \ldots, l_{M-1}\right]: \sum_{m=0}^{M-1} l_{m}=\right.$ $N-t\}, P_{e \mid \boldsymbol{d}_{1}, \boldsymbol{\kappa}, T}\left(\boldsymbol{b}^{m, \pm}, \boldsymbol{l}, t\right)$ is the conditional SER given a transmitted data symbol $\boldsymbol{d}_{1}=\boldsymbol{b}^{m, \pm}$, an interference vector $\boldsymbol{\kappa}=\boldsymbol{l}$, and $T=t$ users in first group. Here $\boldsymbol{b}^{m, \pm}=\left[b_{0}^{m}, b_{1}^{m}, \ldots, b_{M-1}^{m}\right]$ with $b_{m}^{m}= \pm 1$ and $b_{i}^{m}=0$ for any $i \in \mathcal{M}-\{m\}$. Because of the symmetry of the modulation format, the last equation reduces to:

$$
P_{e}=\sum_{t=t_{\min }}^{t_{\max }} \sum_{\boldsymbol{l} \in \mathcal{X}} P_{e \mid \boldsymbol{d}_{\mathbf{1}}, \boldsymbol{\kappa}, T}\left(\boldsymbol{b}^{0,+}, \boldsymbol{l}, t\right) P_{\boldsymbol{\kappa}} T(\boldsymbol{l} \mid t) P_{T}(t)
$$

Using a union bound, the last conditional SER can be upperbounded as:

$$
\begin{aligned}
& P_{e \mid \boldsymbol{d}_{\mathbf{1}}, \boldsymbol{\kappa}, T}\left(\boldsymbol{b}^{0,+}, \boldsymbol{l}, t\right) \\
&= \operatorname{Pr}\left\{I_{0} \leq\left|I_{m}\right|, \text { some } m \in \mathcal{M}-\{0\} \mid \boldsymbol{d}_{\mathbf{1}}=\boldsymbol{b}^{0,+},\right. \\
&\boldsymbol{\kappa}=\boldsymbol{l}, T=t\} \\
& \leq(M-1) \operatorname{Pr}\left\{I_{0} \leq\left|I_{1}\right| \mid \boldsymbol{d}_{\mathbf{1}}=\boldsymbol{b}^{0,+}, \boldsymbol{\kappa}=\boldsymbol{l}, T=t\right\} \\
& \leq(M-1) \operatorname{Pr}\left\{I_{0} \leq I_{1} \mid \boldsymbol{d}_{\mathbf{1}}=\boldsymbol{b}^{0,+}, \boldsymbol{\kappa}=\boldsymbol{l}, T=t\right\} \\
&+(M-1) \operatorname{Pr}\left\{I_{0} \leq-I_{1} \mid \boldsymbol{d}_{\mathbf{1}}=\boldsymbol{b}^{0,+}, \boldsymbol{\kappa}=\boldsymbol{l}, T=t\right\} \\
&= 2(M-1) \operatorname{Pr}\left\{I_{0} \leq I_{1} \mid \boldsymbol{d}_{\mathbf{1}}=\boldsymbol{b}^{0,+}, \boldsymbol{\kappa}=\boldsymbol{l}, T=t\right\}
\end{aligned}
$$

where the last equality is justified by noticing that the mean of $I_{1}$, given $\boldsymbol{d}_{\mathbf{1}}=\boldsymbol{b}^{0,+}$, is zero. Using the Gaussian approximation method, the evaluation of the last probability is immediate:

$$
\begin{aligned}
& P_{e \mid \boldsymbol{d}_{\mathbf{1}}, \boldsymbol{\kappa}, T}\left(\boldsymbol{b}^{0,+}, \boldsymbol{l}, t\right) \\
&= 2(M-1) \operatorname{Pr}\left\{I_{1}-I_{0} \geq 0 \mid \boldsymbol{d}_{\mathbf{1}}=\boldsymbol{b}^{0,+}, \boldsymbol{\kappa}=\boldsymbol{l}, T=t\right\} \\
&= \frac{2(M-1)}{\sqrt{2 \pi\left(\sigma_{0, \boldsymbol{b}^{0,+}, \boldsymbol{l}}^{2}+\sigma_{1, \boldsymbol{b}^{0,+}, \boldsymbol{l}}^{2}\right)}} \\
& \times \int_{0}^{\infty} \exp \left[\frac{\left\{y-\left(\mu_{1, \boldsymbol{b}^{0,+}}-\mu_{0, \boldsymbol{b}^{0,+}}\right)\right\}^{2}}{2\left(\sigma_{0, \boldsymbol{b}^{0,+}, \boldsymbol{l}}^{2}+\sigma_{1, \boldsymbol{b}^{0,+}, \boldsymbol{l}}^{2}\right)}\right] d y \\
&=(M-1) \operatorname{erfc}\left[\frac{\mu_{0, \boldsymbol{b}^{0,+}}-\mu_{1, \boldsymbol{b}^{0,+}}}{\sqrt{2\left(\sigma_{0, \boldsymbol{b}^{0,+}, \boldsymbol{l}}^{2}+\sigma_{1, \boldsymbol{b}^{0,+}, \boldsymbol{l}}^{2}\right)}}\right] \\
&=(M-1) \operatorname{erfc}\left[\frac{\mathcal{R}_{0} w / L}{\sqrt{2\left(\sigma_{0, \boldsymbol{b}^{0,+}, \boldsymbol{l}}^{2}+\sigma_{1, \boldsymbol{b}^{0,+}, \boldsymbol{l}}^{2}\right)}}\right] .
\end{aligned}
$$

Finally, the bit-error rate can be written as

$$
\begin{aligned}
P_{b}= & \frac{M}{2 M-1} P_{e} \\
\leq & \frac{M(M-1)}{2 M-1} \sum_{t=t_{\min }}^{t_{\max }} \sum_{l_{0}=0}^{N-t} \sum_{l_{1}=0}^{N-t-l_{0}} P_{\kappa_{0} \kappa_{1} \mid T}\left(l_{0}, l_{1} \mid t\right) P_{T}(t) \\
& \times \operatorname{erfc}\left[\frac{\mathcal{R} P_{0} w / L}{\sqrt{2\left(\sigma_{0, \boldsymbol{b}^{0,+}, \boldsymbol{l}}^{2}+\sigma_{1, \boldsymbol{b}^{0,+}, \boldsymbol{l}}^{2}\right)}}\right]
\end{aligned}
$$




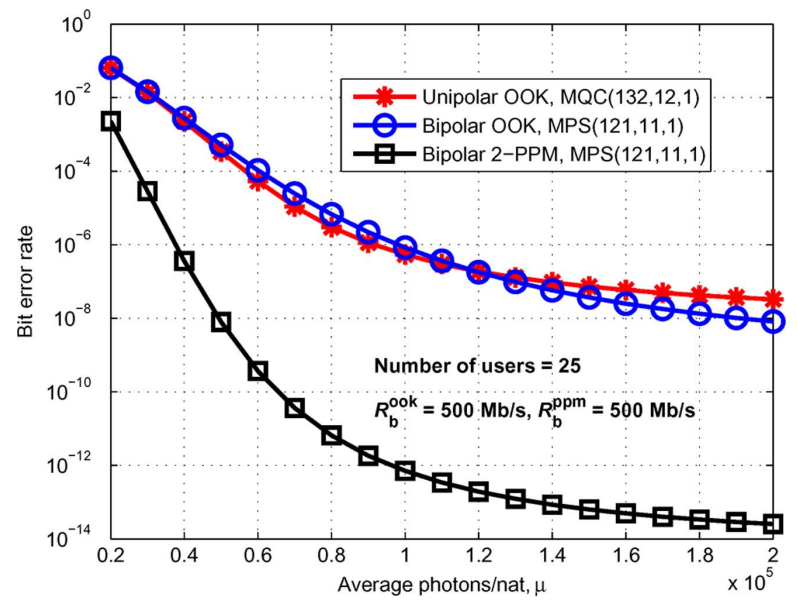

Fig. 2. Bit error rates of SAC-OCDMA receivers versus the average energy per nat for number of active users $N=25$ and a transmission rate $R_{b}=500$ $\mathrm{Mb} / \mathrm{s}$.

where

$$
\begin{aligned}
& P_{\kappa_{0} \kappa_{1}{ }^{\prime} T}\left(l_{0}, l_{1} \mid t\right) \\
& =\frac{(N-t) !}{l_{0} ! l_{1} !\left(N-t-l_{0}-l_{1}\right) !} \\
& \quad \times\left(\frac{1}{M}\right)^{l_{0}+l_{1}}\left(1-\frac{2}{M}\right)^{N-t-l_{0}-l_{1}} .
\end{aligned}
$$

\section{Numerical Results}

In our numerical evaluations we assume that both the receiver bandwidth $B_{e}$ and the average transmitted photons per nat $\mu$ are held fixed. For an ideal channel, the peak single-user received power is thus given by:

$$
P_{0}= \begin{cases}4 \log 2 \eta e B_{e} \mu / \mathcal{R} ; & \text { for Unipolar OOK, } \\ 2 \log 2 \eta e B_{e} \mu / \mathcal{R} ; & \text { for Bipolar OOK, } \\ 2 \log (2 M) \eta e B_{e} \mu / \mathcal{R} ; & \text { for Bipolar MPPM, }\end{cases}
$$

where $\eta$ is the photodiode efficiency. The bit-error rate as given in (27) has been evaluated numerically for a modified prime sequence (MPS) code with length $L=121$ and weight $w=11$ and plotted in Fig. 2 versus different values of average photons per information nat. Other parameters used in the evaluations are as follows. The data bit rate $R_{b}=500 \mathrm{Mb} / \mathrm{s}$, the linewidth of the light source $\Delta \nu=2.5 \mathrm{THz}$, the coherence time $\tau_{c}=48.4$ ps, the pulse-position multiplicity $M=2$, the receiver noise temperature $T^{\circ}=300 \mathrm{~K}$, the receiver load resistance $R_{L}=1$ $\mathrm{k} \Omega$, and the number of simultaneous users $N=25$ users. In addition, the BERs of both unipolar OOK, adopting MQC codes with $L=132$ and $w=12$, and bipolar OOK, adopting modified prime sequence codes with $L=121$ and $w=11$, are also plotted in the same figure for the sake of comparison. The superiority of our proposed technique in clear from the figure. Indeed, the proposed system is more power efficient as only $5 \times 10^{4}$ photons are needed to reach a BER of $10^{-8}$, whereas about $2 \times 10^{5}$ photons are needed to reach same BER for the case

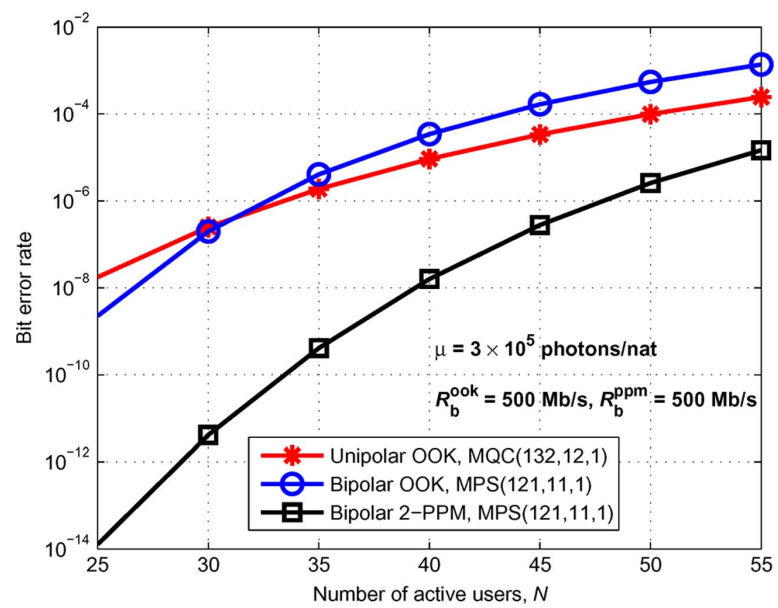

Fig. 3. Bit error rates of SAC-OCDMA receivers versus the number of active users for an average energy per nat $\mu=3 \times 10^{5}$ photons/nat and a transmission rate $R_{b}=500 \mathrm{Mb} / \mathrm{s}$.

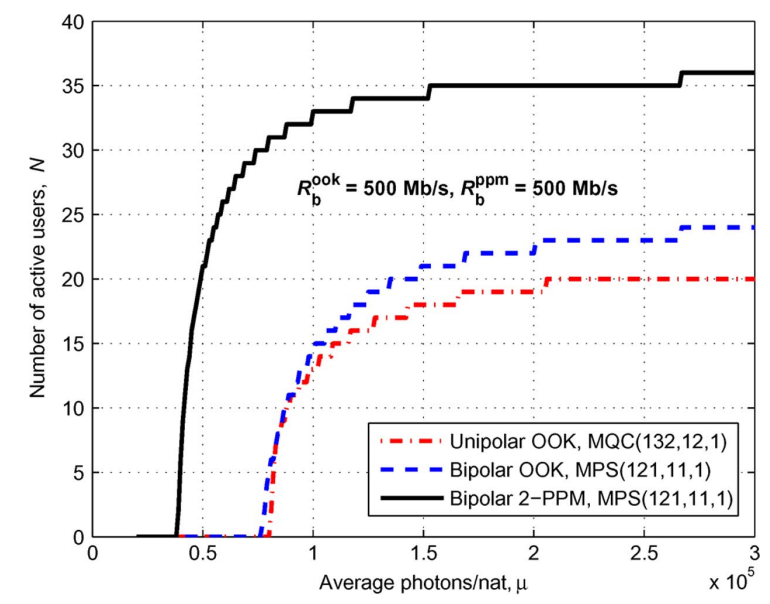

Fig. 4. Comparison between the maximum achievable number of users in SAC-OCDMA receivers versus the average energy per nat for bit error rates not exceeding $10^{-9}$.

of OOK systems. That is, our system saves about $75 \%$ of energy. In addition, it is clear from the figure that other traditional systems cannot achieve higher quality communications, e.g., a BER of $10^{-12}$, without decreasing either the number of simultaneous users or the data rate. However this stringent BER can be achieved easily by our system. This observation is confirmed in Fig. 3, which has same parameters as in Fig. 2 but plotted versus the number of active users for high average photons per nat. It is obvious that at a BER of $10^{-8}$, only about 26 simultaneous users can be loaded in traditional systems, whereas about 40 users can communicate simultaneously in the proposed system. A comparison between the maximum achievable number of users in SAC-OCDMA receivers versus the average photons per nat for BER not exceeding $10^{-9}$ is plotted in Fig. 4, with similar parameters as given in Fig. 2. It is clear from the figure that the proposed system can come across the limitations of traditional systems by providing both energy efficiency and high data rate transmission. Finally in Fig. 5 we give a comparison between the maximum achievable number of users in SAC-OCDMA receivers with bipolar 2-PPM versus the average energy per nat 


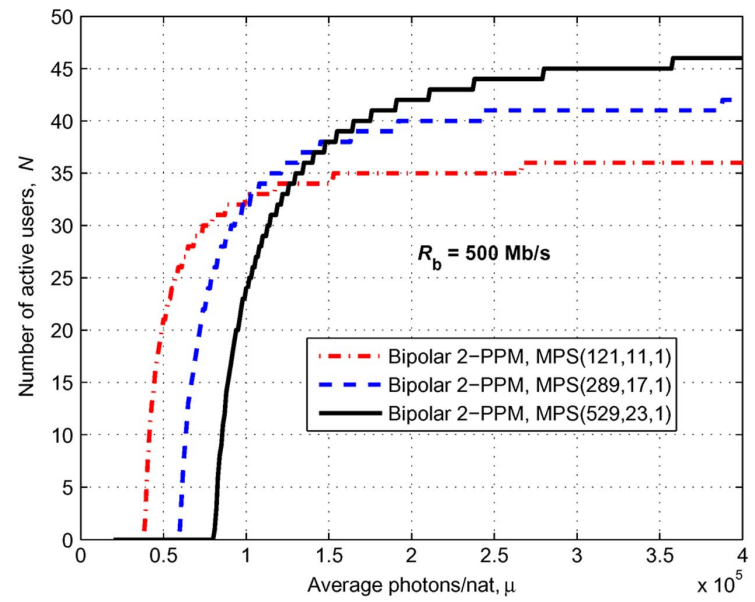

Fig. 5. Comparison between the maximum achievable number of users in SAC-OCDMA receivers with bipolar 2-PPM versus the average energy per nat for different values of prime numbers and bit error rates not exceeding $10^{-9}$.

for different values of prime numbers and bit error rates not exceeding $10^{-9}$. It is clear from the figure that for low number of simultaneous users, it is more efficient to use low prime number for coding, whereas for high number of users, power efficiency increases by increasing the prime number. Notice that when there is an excessive increase in the number of simultaneous users, low prime numbers cannot be achieved. From the above discussion, it is clear that our proposed system is suitable for high data rate transmission, whereas traditional systems cannot achieve high rate with acceptable number of users.

\section{Extension AND Multicode Modulation}

The above technique can be extended to higher levels of $M P P M$ with more-than-two orthogonal code sequences (or multicode modulation). This keeps both the transmission rate and system bandwidth fixed. It can be shown that the bit-error rate for $K$-level $M$ PPM scheme with multicode modulation, $K \in\{2,3, \cdots, \min \{p, p /\lceil N / p\rceil\}\}$, is upper bounded by

$$
\begin{aligned}
P_{b} \leq & \frac{M K^{2}(M-1)}{4(K M-1)} \\
& \times \sum_{t=\max \left\{1, N-(p-1)\left\lfloor\frac{p}{K}\right\rfloor\right\}}^{\min \left\{N,\left\lfloor\frac{p}{K}\right\rfloor\right\} \quad\left(\begin{array}{c}
\left\lfloor\frac{p}{K}\right\rfloor-1 \\
t-1
\end{array}\right)\left(\begin{array}{c}
(p-1)\left\lfloor\frac{p}{K}\right\rfloor \\
N-t
\end{array}\right)} \\
& \times \sum_{l_{0}=0}^{N-t} \sum_{l_{1}=0}^{N-t-l_{0}} P_{\kappa_{0} \kappa_{1} \mid T}\left(l_{0}, l_{1} \mid t\right) \\
& \times \operatorname{erfc}\left[\frac{\mathcal{R} P_{0} w / L}{\sqrt{2\left(\sigma_{0, \boldsymbol{b}^{0,+}, \boldsymbol{l}}^{2}+\sigma_{1, \boldsymbol{b}^{0,+}, \boldsymbol{l}}^{2}\right)}}\right]
\end{aligned}
$$

where for $K$-level $M \mathrm{PPM}$, the received power and transmission rate are modified to:

$$
\begin{aligned}
P_{0} & =\frac{2 \log (K M) \eta e B_{e} \mu}{\mathcal{R}} \\
R_{b} & =\frac{\log (K M)}{M \tau}
\end{aligned}
$$

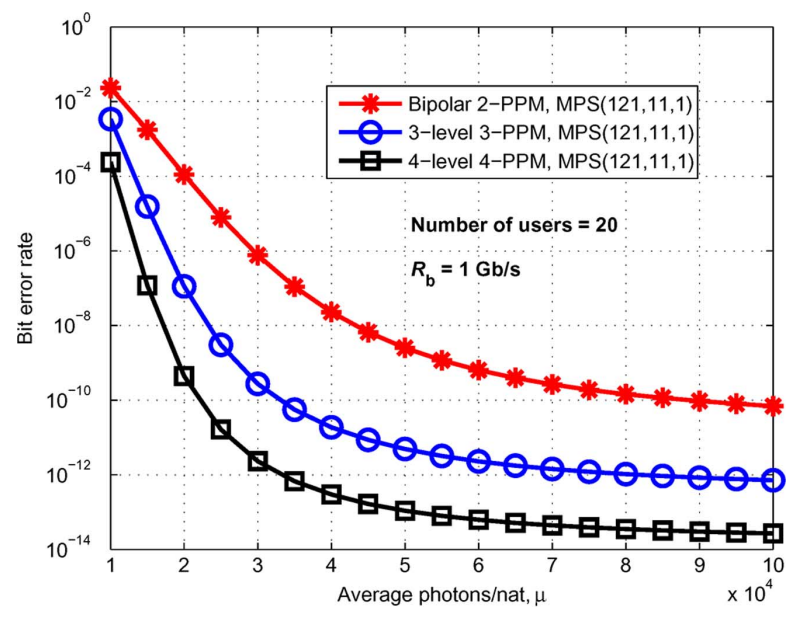

Fig. 6. Bit error rates of SAC-OCDMA receivers of $K$-level $M$ PPM schemes with multicode modulation versus the average energy per nat for number of active users $N=20$, a transmission rate $R_{b}=1 \mathrm{~Gb} / \mathrm{s}$, and pulse-position multiplicity $M \in\{2,3,4\}$.

respectively. In order to keep the rate not less that of OOK, we choose $K=\left\lceil\left(2^{M}\right) /(M)\right\rceil$. A BER comparison for $K$-level $M$ PPM scheme with multicode modulation, as give in (30), is shown in Fig. 6 for transmission rate of $1 \mathrm{~Gb} / \mathrm{s}$ and $M \in$ $\{2,3,4\}$. The improvement with higher values of $M$ is clear from the figure. The price to be paid, however, is the increase in detection complexity.

\section{CONCLUSION}

A modulation technique has been proposed for SACOCDMA systems in order to further reduce the effect of phase-induced intensity noise. This technique adopts modified prime sequence codes and assigns two orthogonal code sequences to each user. Data symbols from a user are modulated using two-level $M$-ary pulse-position modulation (MPPM) scheme, each level would correspond to a different code sequence. The bit error rate (BER) of the proposed system has been derived and compared numerically to that of corresponding systems adopting complementary and non-complementary keying. In addition, achievable number of users of the proposed and traditional techniques are compared. It turned out that the proposed system is more power efficient than traditional techniques and more suitable for reliable high data rate transmission with suitable number of simultaneous users. Extension to higher levels of MPPM schemes with multicode modulation has been studied as well.

\section{REFERENCES}

[1] D. Zaccarin and M. Kavehrad, "An optical CDMA system based on spectral encoding of LED," IEEE Photon. Technol. Lett., vol. 4, pp. 479-482, Apr. 1993.

[2] D. Zaccarin and M. Kavehrad, "Performance evaluation of optical CDMA systems using non-coherent detection and bipolar codes," $J$ Lightw. Technol., vol. 12, pp. 96-105, Jan. 1994.

[3] T. A. Bhuiyan, M. Z. Hassan, S. M. S. Tanzil, S. Hayder, and S. P. Majumder, "Performance improvement of IM-DD free space optical CDMA (attenuated by strong atmospheric turbulence) with maximal ratio combining," in Proc. IEEE Int. Conf. Comput. Intel. Commun. Net. (CICN 2010), Bhopal, India, Nov. 26-28, 2010, pp. 513-518. 
[4] S.-P. Tseng and J. Wu, "The SAC OCDMA PON with super perfect difference codes," in Proc. IEEE Int. Conf. Commun. (ICC 2011), Kyoto, Japan, June 5-9, 2011, pp. 1-4.

[5] C. Y. Chang, G. C. Yang, C. Y. Chang, and W. C. Kwong, "Study of a diversity O-CDMA scheme for optical wireless," J. Lightw. Technol., vol. 30, pp. 1549-1558, May 15, 2012.

[6] E. D. J. Smith, R. J. Blaikie, and D. P. Taylor, "Performance enhancement of spectral-amplitude-coding optical CDMA using pulse-position modulation," IEEE Trans. Commun., vol. 46, no. 9, pp. 1176-1185, Sept. 1998.

[7] X. Zhou, H. M. H. Shalaby, and C. Lu, "Design and performance analysis of a new code for spectral-amplitude-coding optical CDMA systems," in Proc. IEEE 6th Int. Symp. Spread Spectrum Tech. Applications (ISSSTA 2000), Sep. 6-8, 2000, pp. 174-178.

[8] Z. Wei, H. M. H. Shalaby, and H. Ghafouri-Shiraz, "Modified quadratic congruence codes for fiber Bragg-grating-based spectral-amplitude-coding optical CDMA systems," J. Lightw. Technol., vol. 19 , no. 9, pp. 1274-1281, Sep. 2001.

[9] Z. Wei and H. Ghafouri-Shiraz, "Proposal of a novel code for spectral amplitude-coding optical CDMA systems," IEEE Photonics Technol. Lett., vol. 14, no. 3, pp. 414-416, Mar. 2002.

[10] Z. Wei and H. Ghafouri-Shiraz, "Codes for spectral-amplitude-coding optical CDMA systemss," J. Lightw. Technol., vol. 20, no. 8, pp. 1284-1291, Aug. 2002.

[11] W. C. Kwong, P. A. Perrier, and P. R. Prucnal, "Performance comparison of asynchronous and synchronous code-division multiple-access," IEEE Trans. Commun., vol. COM-39, pp. 1625-1634, Nov. 1991.

[12] N. T. Dang, H. T. T. Pham, and A. T. Pham, "Novel 2-D OCDMA systems using multi-code modulation and optical hard-limiters," in Proc. 3rd Int. Conf. Commun. Elect. (ICCE 2010), Nha Trang, Vietnam, Aug. 11-13, 2010, pp. 242-246.

[13] N. T. Dang, H. T. T. Pham, and A. T. Pham, "Novel multi-code pulse position modulation for performance improvement of 2-D OCDMA systems," in Proc. 17th Asia-Pacific Conf. Commun. (APCC 2011), Kota Kinabalu, Sabah, Malaysia, Oct. 2-5, 2011, pp. 96-101.

[14] J. Magné, D.-P. Wei, S. Ayotte, L. A. Rusch, and S. LaRochelle, "Experimental demonstration of frequency-encoded optical CDMA using superimposed fiber Bragg gratings," in Proc. Conf. Bragg Gratings, Poling \& Photosensitivity in Glass Waveguides (BGPP 2003), Monterey, California, Sept. 1, 2003, pp. 294-296.

[15] S. Ayotte, M. , J. Magné, L. A. Rusch, and S. LaRochelle, "Experimental verification and capacity prediction of FE-OCDMA using superimposed FBG," J. Lightw. Tech., vol. 23, no. 2, pp. 724-731, Feb. 2005.
[16] M. Noshad and K. Jamshidi, "Bounds for the BER of codes with fixed cross correlation in SAC-OCDMA systems," J. Lightw. Tech., vol. 29, no. 13 , pp. 1944-1950, July 2011.

[17] H. M. H. Shalaby, "Closed form expression for the bit-error rate of spectral-amplitude-coding optical CDMA systems," IEEE Photon. Technol. Lett., vol. 24, pp. 1285-1287, Aug. 1, 2012.

[18] M. M. Rad and J. A. Salehi, "Phase-induced intensity noise in digital incoherent all-optical tapped-delay line systems," J. Lightw. Tech., vol. 24, no. 8, pp. 3059-3072, Aug. 2006.

Hossam M. H. Shalaby (S'83-M'91-SM'99) was born in Giza, Egypt, in 1961. He received the B.S. and M.S. degrees from Alexandria University, Alexandria, Egypt, in 1983 and 1986, respectively, and the Ph.D. degree from the University of Maryland at College Park in 1991, all in electrical engineering.

In 1991, he joined the Electrical Engineering Department, Alexandria University, and was promoted to Professor in 2001. Currently he is on leave from Alexandria University, where he is the chair of the Department of Electronics and Communications Engineering, School of Electronics, Communications, and Computer Engineering, Egypt-Japan University of Science and Technology (E-JUST), New Borg EL-Arab City, Alexandria, Egypt. From December 2000 to 2004, he was an Adjunct Professor with the Faculty of Sciences and Engineering, Department of Electrical and Information Engineering, Laval University, Quebec, QC, Canada. From September 1996 to February 2001, he was on leave from the Alexandria University. From September 1996 to January 1998, he was with the Electrical and Computer Engineering Department, International Islamic University Malaysia, and from February 1998 to February 2001, he was with the School of Electrical and Electronic Engineering, Nanyang Technological University, Singapore. His research interests include optical communications, optical CDMA, optical burst-switching, OFDM technology, and information theory.

Dr. Shalaby has served as a student branch counselor at Alexandria University, IEEE Alexandria and North Delta Subsection, from 2002 to 2006, and served as a chairman of the student activities committee of IEEE Alexandria Subsection from 1995 to 1996 . He received an SRC fellowship from 1987 to 1991 (from Systems Research Center, Maryland; State Excellence Award in Engineering Sciences in 2007 from Academy of Scientific Research and Technology, Egypt), Shoman Prize for Young Arab Researchers in 2002 from The Abdul Hameed Shoman Foundation, Amman, Jordan; the State Incentive Award in Engineering Sciences both in 1995 and 2001 from the Academy of Scientific Research and Technology, Egypt; the University Excellence Award in 2009 from Alexandria University; and the University Incentive Award in 1996 from Alexandria University. 